\title{
Ácido Láctico como Indicativo de Aptidão Física em Ratos
}

\author{
Lactic Acid as an Indication of Physical Fitness in Rats
}

Artigo Original

Honório Sampaio Menezes? Julio Cezar Dors Coracini² Kellyn Cristine Kepler ${ }^{3}$ Elemara Frantz $z^{4}$ Milena Pacheco Abegg ${ }^{5}$ Cora Albrecht Correa ${ }^{6}$ Silvia Letícia Merceo Bacchi Cirino

1. Professor Adjunto-Doutor do Curso de Medicina da Universidade Luterana do Brasil (Ulbra) e da Fundação Universitária de Cardiologia/Instituto de Cardiologia, Porto Alegre, RS, Brasil.

2. Acadêmico de Medicina da Ulbra.

3. Fisioterapeuta, Mestranda

da Fundação Universitária de Cardiologia/Instituto de Cardiologia, Porto Alegre, RS, Brasil.

4. Enfermeira, Mestranda da

Fundação Universitária de

Cardiologia/Instituto de Cardiologia, Porto Alegre, RS, Brasil.

5. Bolsista ICFAPERGS, Acadêmica de Medicina membro do Grupo de Pesquisa em Medicina Experimental da Ulbra.

6. Bolsista ProlCUlbra, Acadêmica de Medicina membro do Grupo de Pesquisa em Medicina Experimental da Ulbra.

7. Acadêmica de Medicina membro do Grupo de Pesquisa em Medicina Experimental da Ulbra.

Endereço para correspondência: Rua D. Pedro II, 1.220 Cj. 316 Higienópolis - 90550-141 - Porto Alegre, RS

E-mail:

hsmenezes@computech.com.br

\begin{abstract}
RESUMO
Introdução: Os modelos experimentais são utilizados para simulações de situações relacionadas ao treinamento físico. Objetivo: O objetivo do presente estudo foi verificar a aptidão física de ratos em treinamento através da avaliação das concentrações de ácido láctico sérico. Metodologia: Foi realizado estudo experimental utilizando 40 ratos machos Wistar, adultos, divididos em cinco grupos de oito indivíduos, havendo um grupo controle. Foi realizado o primeiro teste de esforço máximo (TE) após uma semana de familiarização com a esteira rolante e antes do primeiro dia de treinamento. Os testes de esforço seguintes foram feitos a cada 10 dias de treinamento. No final de cada teste de esforço foi coletado $1 \mathrm{ml}$ de sangue para medir o ácido láctico. $O$ teste $t$ de Student foi utilizado para comparação das médias. Resultados: O ácido láctico sérico diminuiu significativamente entre o primeiro e o último teste de esforço máximo nos grupos GE10, 20 e 30. No G30 pode-se demonstrar diferença significativa entre os testes inicial e final no que se refere à velocidade $(p=0,003)$ e tempo $(p=0,018)$, assim como no $G 40$ velocidade inicial $(p=0,0006)$ e tempo $(p=0,0001)$. Conclusão: Os resultados demonstraram que ratos submetidos a treinamento físico apresentam capacidade metabólica medida pelo ácido láctico, que demonstra aptidão física e treinamento adequado com aumento do esforço físico, sendo bem tolerado até 40 dias de treinamento em esteira rolante.
\end{abstract}

Palavras-chave: rato, exercício físico, ácido láctico.

\begin{abstract}
Introduction: Experimental models are used for simulation of situations related to physical training. Objective: This study had the objective of verifying the fitness of rats in training; this was achieved through the measurement of the concentration of lactic acid in the blood serum. Methodology: An experimental study using 40 Wistar male adult rats, divided into five groups of eight individuals and a control group was carried out. The first test of Maximum Effort (TE) was applied after one week of familiarization with the treadmill running and right before the first day of training. The following effort tests were applied at every ten days. In order to measure the concentration of lactic acid in the blood after the test; it $1 \mathrm{ml}$ of blood was collected from each rat. The Student $t$ test was used to compare the means. Results: Lactic acid serum significantly decreased in groups GE10, 20, 30 between the first and the last effort test. In the $\mathrm{G} 30$ there was a significant difference between the initial and final tests regarding speed $(p=0.003)$ and time $(p=0.018)$, as well as in the G40 group, where the initial velocity was $(p=0.0006)$ and time $(p=0.0001)$. Conclusion: The results measured through the lactic acid in the blood showed that rats submitted to physical training present better metabolic capacity and that with suitable training, the physical fitness increased after the physical effort. This effort is well tolerated by the rats up to 40 days of treadmill running.
\end{abstract}

Keywords: rat, exercise, lactic acid. 


\section{INTRODUÇÃO}

O exercício físico caracteriza-se, principalmente, pela contração do músculo esquelético, cujo gasto de energia denominou-se equivalente metabólico (MET). A energia é acumulada em forma de adenosina trifosfato (ATP); a quebra de ATP do músculo fornece energia para exercícios de diferentes intensidades e durações ${ }^{(1)}$.

A via anaeróbia alática é representada pela quebra da creatinafosfatase (CP), liberando energia três vezes mais do que a ATP, mas proporciona, no máximo, 10 segundos de contração. Caracteriza-se pela rapidez de ressíntese e pela sua utilidade em momentos iniciais de esforços curtos e máximos ${ }^{(2)}$.

A via aeróbia utiliza a glicose, o ácido graxo e os aminoácidos para síntese de energia. A glicólise é o processo promovido pelos citocromos, que constituem o sistema de transporte de elétrons (STE). Enquanto houver equilíbrio entre a produção de elétrons e o aporte de oxigênio, o metabolismo é predominantemente aeróbio e o ácido pirúvico é o produto final desse processo. Por tratar-se de uma via de ativação lenta, necessitando de ativação de inúmeros sistemas enzimáticos e aporte de oxigênio, torna-se responsável pelos exercícios de longa duração(3).

Na realização de atividades físicas de grande intensidade e por um tempo superior ao proporcionado pela via CP, a demanda metabólica aumenta, com isso, a quantidade de elétrons liberada se sobrepõe à capacidade que o NAD apresenta de transportar esses elétrons para o oxigênio e processá-los no STE. Portanto, há altas concentrações de NADH e piruvato, com baixo aporte de oxigênio. Grande parte do ácido pirúvico irá fornecer energia através de uma via alternativa chamada anaeróbia lática, este, no sarcoplasma, sofrerá transformação em ácido láctico, reação catalisada pela enzima lactato desidrogenase $(\mathrm{LDH})^{(3)}$.

Durante o exercício intenso há um aumento da ação da via lática, chegando ao ponto do limiar anaeróbio desencadeando fadiga muscular ${ }^{(4)}$.

O ATP para liberar energia é decomposto em ADP e fosfato e lança um próton, esse é usado na via aeróbia quando há oxigênio suficiente no organismo. No exercício intenso, aumenta a produção de ATP pelas vias não oxidativas, a glicólise e a fosfogênica, para o suporte da contração muscular. Com isso, como não há oxigênio, o próton que foi lançado acumula-se, originando acidose metabólica. A produção de lactato aumenta nessas condições celulares impedido o acúmulo de piruvato, fornecendo NAD para a glicólise ${ }^{(5)}$, este aumento de lactato coincide com a acidose celular e permanece um bom marcador indireto das condições metabólicas da célula que induz a acidose metabólica ${ }^{(5)}$.

O ácido láctico é produzido constantemente no organismo, tendo sua concentração aumentada nos músculos durante atividade física de alta intensidade ${ }^{(6)}$, caracterizando fisiologicamente um estado a partir do qual ocorre desequilíbrio de metabólitos, os quais podem gerar fadiga muscular. Pesquisas têm sido realizadas para desenvolver metodologias capazes de mensurar a intensidade do esforço correspondente ao limiar de lactato (LL), ou limiar anaeróbio (Lan) ${ }^{(7,8)}$, ponto de onde o lactato começa a ser acumulado no sangue ou a intensidade do exercício em que a concentração de lactato aumenta abruptamente ${ }^{(9)}$. Ascensão et al. afirmam que a carga de trabalho correspondente ao $L L$ é a mais elevada sustentada, predominantemente, pelo metabolismo aeróbio ${ }^{(10)}$, conceito que pode variar entre autores ${ }^{(11)}$. Assim, a máxima fase estável do lactato (MFEL) é utilizada para determinar a intensidade de um exercício contínuo na qual ocorre a transição da predominância metabólica aeróbia para anaeróbia ${ }^{(12)} \mathrm{O}$ que significa a maior intensidade de um exercício contínuo na qual a concentração de lactato sanguíneo deixa de aumentar ${ }^{(13)}$. Heck et al. definem o MFEL como a razão entre o lactato transportado no sangue e o lactato removido a partir do sangue ${ }^{(14)}$. Svedahl e McIntosh consideram MFEL como sendo a condição em que o lactato não é acumulado e que a captação de oxigênio pode suprir as necessidades metabólicas decorrentes do exercício realizado ${ }^{(15)}$. A velocidade de lactato mínimo (VLM) é a velocidade na qual é mensurado o menor valor de lactato sanguíneo antes do início da acidose metabólica ${ }^{(15)}$.

O limiar de lactato é a intensidade do exercício associada ao aumento do lactato sanguíneo, em geral passa de $1 \mathrm{mM}$ (repouso) para 2 ou $4 \mathrm{mM}$ na primeira carga de trabalho de um teste incremental ${ }^{(15)}$. O lactato sanguíneo correspondente a $4 \mathrm{mM}$ é denominado OBLA (Onset of Blood Lactate Accumulation) e definido como a intensidade do exercício realizado durante o teste incremental para atingir esse valor ${ }^{(16)}$. O limiar de lactato é um marcador de efeitos adversos, tais como habilidade de tolerar o exercício, sobretudo o dinâmico, e a fadiga muscular ${ }^{(17)}$. Por isso, a detecção da predominância metabólica é muito importante para a correta prescrição de exercício a ser realizado ${ }^{(8)}$. Sendo assim, a lactacidemia venosa serve de marcador de aptidão física (cardiorrespiratória), delimitando a intensidade do exercício e a capacidade de trabalho ${ }^{(17,18)}$.

A taxa de crescimento do lactato sanguíneo tem duas fases usadas para prescrições de treinamento, seja aeróbio e/ou anaeróbio, juntamente com suas respectivas intensidades de trabalho ${ }^{(19)}$, para predição da performance em atividades de endurance ${ }^{(20)}$, para avaliar efeitos de treinamento aeróbio(10) e para avaliação da função cardiorrespiratória máxima e da reserva funcional(21), embora a concentração de lactato sanguíneo pode nem sempre ser representativa da concentração intramuscular(22).

Visto que há necessidade de modelo padronizado para treinamento físico para ratos, utilizando esteira rolante, em que possa haver o tempo necessário para mudança fisiológica, para que trabalhos que necessitem da atividade física como meio de pesquisa possam ter um padrão estabelecido, foi testado o presente protocolo.

Assim, o objetivo do presente estudo foi verificar a aptidão física de ratos em treinamento através da avaliação das concentrações de ácido láctico sérico.

\section{METODOLOGIA}

Foi realizado estudo experimental utilizando uma população de 40 ratos machos Wistar, adultos com 250-300g, em ambiente controlado, divididos em cinco grupos:

Grupo Controle: oito ratos adultos sedentários.

Grupo Exercício:

Grupo GE10: 10 dias de exercícios com oito ratos adultos.

Grupo GE20: 20 dias de exercício com oito ratos adultos.

Grupo GE30: 30 dias de exercício com oito ratos adultos.

Grupo GE40: 40 dias de exercício com oito ratos adultos.

Os animais foram mantidos em gaiolas coletivas com oferta de água e comida (ração Nuvital, Nuvilab, Colombo, PR) à vontade, em ambiente na temperatura de 22 a $24^{\circ} \mathrm{C}$ e com ciclos claro e escuro de $12 \mathrm{~h}$ cada.

Durante todo o estudo, observou-se o cumprimento da Lei 6.638/79 e das normas do Manual para Técnicos em Bioterismo, que regulamentam a prática didático-científica e os cuidados mínimos e dignos para animais de experimentação, com eutanásia dos animais através de inalação de $\mathrm{CO}_{2}$. O protocolo foi aprovado pelo Comitê de Ética em Pesquisa do Instituto de Cardiologia do Rio Grande do Sul.

\section{Procedimentos}

Foi realizado o primeiro teste de esforço máximo preliminar (TE) após uma semana de familiarização com a esteira rolante e antes do primeiro dia de treinamento físico para os seguintes grupos: GE10, 
GE20, GE30 e GE40. Os testes de esforço seguintes foram feitos a cada 10 dias de treinamento físico conforme o período de exercício com o objetivo de ajustar as intensidades do treinamento. No final de cada teste de esforço foi coletado $1 \mathrm{ml}$ de sangue na região da cauda após uma pequena incisão em sua extremidade, para medir o ácido láctico (em mg/dL - taxa de conversão para mM usada 0,111).

\section{Cálculo para o treinamento}

Após o teste de esforço máximo, foi obtida a distância média percorrida, e o tempo médio de duração do teste de todos os ratos. Foi calculada, assim, a velocidade média de todos os ratos, através da fórmula Velocidade média = Distância percorrida / Tempo. A fim de obter um treinamento que correspondesse, em média, a 70\% da capacidade de esforço máximo de todos os ratos, foi mantida uma das variáveis da fórmula acima constante, e alterada a outra para $70 \%$ do valor médio obtido no teste de esforço.

\section{Exemplo}

A distância média do teste foi de 190,7 metros (0,1907km) e o tempo médio de $13 \mathrm{~min} 30 \mathrm{~s}(0,225 \mathrm{~h})$, a velocidade média seria de $0,85 \mathrm{~km} / \mathrm{h}$. Para obter um treinamento com $70 \%$ de intensidade, pode-se manter o tempo de 13min30s constante, e alterar a distância percorrida para 70\% de 190,7m (134m). Assim, a velocidade média do exercício passa a ser $0,134 \mathrm{~km} / 0,225 \mathrm{~h}$, que é igual a 0,6km/h. Essa velocidade corresponde a $70 \%$ da velocidade média do teste $(0,85 \mathrm{~km} / \mathrm{h})$.

No treinamento foi mantido o tempo constante e diminuída a distância percorrida para 70\% do valor do teste, com consequente diminuição da velocidade média do treinamento, também em 70\%.

Como o teste de esforço foi realizado com variação progressiva de velocidade, o treinamento também foi realizado com variação de velocidade. O tempo definido para o treinamento foi dividido em cinco fases: aquecimento, aceleração, pico máximo, desaceleração e desaquecimento, buscando obter melhores resultados. Veja no quadro 1 que o tempo (13min30s) foi dividido nas cinco fases, e a velocidade de cada fase foi calculada de forma que a distância total percorrida fosse de aproximadamente 134 metros (70\%). Procurou-se também fazer as variações de velocidade de $0,3 \mathrm{~km}$, à semelhança do teste de esforço, de forma que os ratos acostumassem com incrementos de velocidade sempre idênticos. Ainda, na fase "pico máximo", foi utilizada velocidade de $70 \%$ da velocidade máxima atingida, em média, pelos ratos no teste de esforço.

Quadro 1. Exemplificando o protocolo de treinamento.

\begin{tabular}{|c|c|c|c|c|c|}
\hline Tempo & $3 \mathrm{~min}$ & $2 \mathrm{~min}$ & $3 \mathrm{~min}$ & $2 \mathrm{~min}$ & $3,5 \mathrm{~min}$ \\
\hline Velocidade & $0,4 \mathrm{~km} / \mathrm{h}$ & $0,7 \mathrm{~km} / \mathrm{h}$ & $1,0 \mathrm{~km} / \mathbf{h}$ & $0,7 \mathrm{~km} / \mathrm{h}$ & $0,4 \mathrm{~km} / \mathrm{h}$ \\
\hline Cap. máx. & $27 \%$ & $47 \%$ & $70 \%$ & $47 \%$ & $27 \%$ \\
\hline & \multicolumn{2}{|c|}{$37 \%$ tempo total } & $22 \%$ tempo total & \multicolumn{2}{|c|}{$41 \%$ tempo total } \\
\hline
\end{tabular}

A cada 10 dias esse teste era refeito e o treinamento ajustado de acordo com a nova capacidade dos ratos.

O grupo controle não realizou teste de esforço, o sangue foi coletado em repouso no primeiro dia do estudo e após 40 dias, dosando-se a concentração de ácido láctico.

Todos os grupos foram comparados nos primeiros 10 dias, porém, com a eliminação do GE10, a segunda comparação foi feita somente com os grupos GE20, GE30, GE40 e GC e assim sucessivamente.

No último dia de treinamento de cada grupo, o resultado do primeiro teste de esforço foi condicionado ao rato. Ao término deste, o sangue foi coletado. Esse procedimento foi realizado para avaliar o
GRUPO EXERCÍCIO

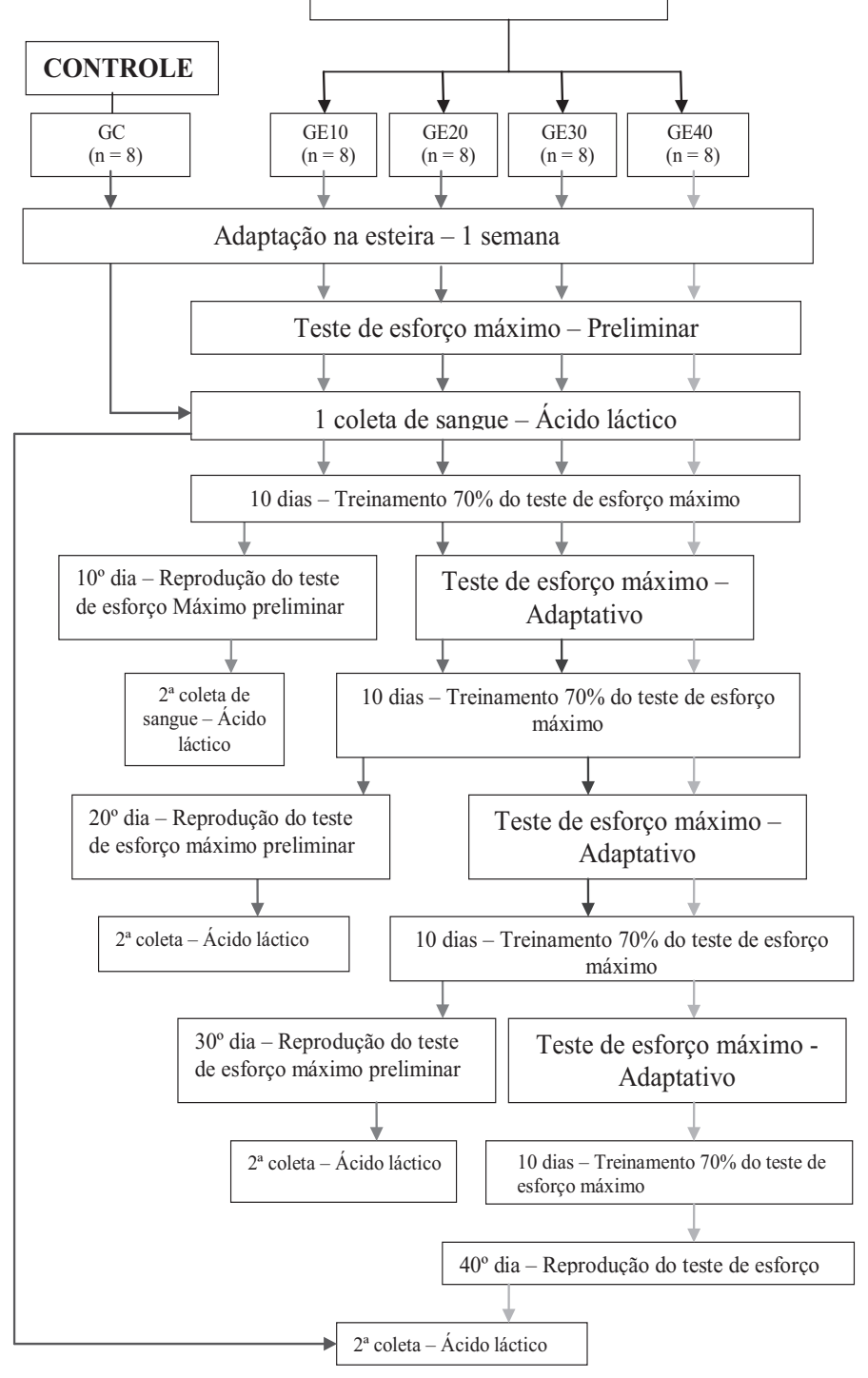

Figura 1 - Fluxograma para o Modelo Experimental Wistar

ácido láctico inicial (primeiro teste de esforço) e o ácido láctico final repetindo os mesmos valores do primeiro teste averiguando se houve ou não alguma mudança.

\section{Análise estatística}

Os resultados foram expressos utilizando estatística descritiva (média \pm desvio padrão). A comparação entre as médias dos testes de esforço foi através do teste $t$ de Student. O limite alfa considerado foi de 5\%, com nível de significância de 0,05.

\section{RESULTADOS}

Os resultados das avaliações estão expressos nas tabelas 1 e 2 abaixo relacionadas.

Na tabela 1 percebe-se que o ácido láctico sérico diminuiu significativamente entre o esforço máximo inicial do primeiro teste e sua reprodução realizada no último dia de treinamento nos grupos GE10, 20 e 30. O grupo GE40 não apresentou diferença entre os testes. O grupo controle aumentou o ácido láctico na segunda coleta, a qual foi realizada 40 dias após a primeira.

$\mathrm{Na}$ tabela 2 pode-se perceber que os ratos correram mais rápido e por mais tempo após 20 dias de treinamento, havendo diferença estatística de tempo e de velocidade (grupo 30 e grupo 40). 
Tabela 1. Médias e desvios padrão do nível de ácido láctico sérico (mg/dl) por grupo de ratos, coletado após esforço máximo inicial no primeiro dia de treinamento e no último dia de treinamento após a reprodução do esforço máximo inicial e a diferença percentual entre as medidas.

\begin{tabular}{c|c|c|c|c}
\hline Grupos & $\begin{array}{c}\text { Esforço } \\
\text { máximo inicial }\end{array}$ & $\begin{array}{c}\text { Reprodução } \\
\text { do esforço } \\
\text { máximo inicial }\end{array}$ & $\begin{array}{c}\text { Diferença } \\
\text { percentual entre } \\
\text { as medidas }\end{array}$ & $\mathbf{p}$ \\
\hline GE10 & $\begin{array}{c}53,37(5,9) \\
( \pm 8,78)\end{array}$ & $\begin{array}{c}25,30(2,8) \\
( \pm 6,39)\end{array}$ & $52,59 \%$ & 0,01 \\
\hline GE20 & $\begin{array}{c}62,13(6,9) \\
( \pm 4,68)\end{array}$ & $\begin{array}{c}43,87(4,8) \\
( \pm 6,50)\end{array}$ & $29,40 \%$ & 0,006 \\
\hline GE30 & $\begin{array}{c}57,00(6,3) \\
( \pm 15,14)\end{array}$ & $\begin{array}{c}32,47(3,6) \\
( \pm 4,30)\end{array}$ & $43,04 \%$ & 0,05 \\
\hline GE40 & $\begin{array}{c}44,47(4,9) \\
( \pm 10,1)\end{array}$ & $\begin{array}{c}40,70(4,5) \\
( \pm 5,96)\end{array}$ & $8,47 \%$ & 0,25 \\
\hline GC & $\begin{array}{c}11,20(1,2) \\
( \pm 3,24)\end{array}$ & $\begin{array}{c}28,97(3,2) \\
( \pm 9,11)\end{array}$ & $158,63 \%$ & 0,03 \\
\hline
\end{tabular}

\pm desvio padrão; $p=$ valor segundo teste $t$ Student (conversão em $\mathrm{mM} / \mathrm{L}$ ).

GC (grupo controle) $=$ coletado ácido láctico em repouso no primeiro dia e após 40 dias.

Tabela 2. Médias e desvios padrão dos testes de esforço máximo por grupo de ratos, referente ao tempo (minutos) e à velocidade (metros/minutos) aplicada, coletados no primeiro dia e no último dia de treinamento.

\begin{tabular}{c|c|c|c|c|c|c|c|c|c}
\hline & GC & G10i & G10f & G20i & G20f & G30i & G30f & G40i & G40f \\
\hline $\begin{array}{c}\text { Tempo } \\
\text { (min) }\end{array}$ & 0 & $\begin{array}{c}13,21 \\
\pm 0,75\end{array}$ & $\begin{array}{c}13,21 \\
\pm 0,75\end{array}$ & $\begin{array}{c}12,91 \\
\pm 1,69\end{array}$ & $\begin{array}{c}13,66 \\
\pm 1,41\end{array}$ & $\begin{array}{c}14,39 \\
\pm 1,17\end{array}$ & $\begin{array}{c}16,40 \\
\pm 1,29\end{array}$ & $\begin{array}{c}13,40 \\
\pm 1,37\end{array}$ & $\begin{array}{c}17,80 \\
\pm 2,04\end{array}$ \\
\hline $\begin{array}{c}\text { Velocidade } \\
\text { (m/min) }\end{array}$ & 0 & $\begin{array}{c}25,00 \\
\pm 0\end{array}$ & $\begin{array}{c}25,00 \\
\pm 0\end{array}$ & $\begin{array}{c}25,00 \\
\pm 2,66\end{array}$ & $\begin{array}{c}26,33 \\
\pm 2,33\end{array}$ & $\begin{array}{c}26,83 \\
\pm 2,66\end{array}$ & $\begin{array}{c}31,33 \\
\pm 2,33\end{array}$ & $\begin{array}{c}25,00 \\
\pm 2,66\end{array}$ & $\begin{array}{c}33,16 \\
\pm 4,50\end{array}$ \\
\hline
\end{tabular}

No G20 não houve diferença na velocidade entre o teste inicial e final $(p=0,753)$, o que aconteceu também com o tempo $(p=0,293)$.

No G30 demonstra-se diferença significativa entre os testes inicial e final no que se refere à velocidade $(p=0,003)$ e tempo $(p=0,018)$.

No G40 percebe-se que a velocidade final aumentou significativamente em relação à velocidade inicial $(p=0,0006)$ e que o tempo também teve diferença significativa $(p=0,0001)$.

\section{DISCUSSÃO}

A determinação com grande acurácia da intensidade do exercício é de suma importância. Há anos as pesquisas não são exclusivas a seres humanos. Os modelos experimentais são fonte de pesquisa interessante para observação do organismo frente ao esforço. Além disso, pode haver simulações de situações fisiopatológicas ou relacionadas ao treinamento que ocorrem nos homens e, com os resultados obtidos, pode-se tentar solucionar, dependendo do acontecimento, os problemas decorrentes das alterações observadas ${ }^{(8)}$. No presente estudo foi utilizado um modelo com ratos e esteira rolante por apresentar determinadas facilidades e não existir um modelo bem determinado para esta condição, evitando-se, assim, emprego de modelo utilizando natação (também usado em muitos experimentos).

O lactato sanguíneo foi identificado como indicador do aumento do metabolismo anaeróbio durante o exercício no início do século passado. Posteriormente, foi usado para a avaliação e a prescrição do treinamento físico de seres humanos. Recentemente, o lactato sanguíneo foi usado para a determinação da intensidade de exercício em animais, mas, apesar da importância, há poucos estudos neste âmbito em ratos ${ }^{(23)}$. Na tabela 1 percebe-se que o lactato medido durante $\mathrm{o}$ esforço máximo inicial (até a exaustão do animal) foi maior do que o medido no esforço final, indicando adaptação metabólica ao exercício na medida em que o treinamento era efetuado, o que também é demonstrado pelo tempo e velocidade de corrida (tabela 2).

Existe uma lacuna na literatura referente a protocolos de avaliação em animais; pesquisas tentam desenvolver métodos para identificar de maneira precisa a intensidade individual de exercício para ratos submetidos a esforços em distintos ergômetros, adaptando à avaliação nos animais em testes já aplicados a humanos. O protocolo considerado padrão ouro para a identificação da transição metabólica aeróbia/ anaeróbia durante exercício, tanto no homem como no animal, é o de máxima fase estável de lactato (MFEL). A MFEL representa a intensidade mais elevada na qual ocorre estabilização do lactato sanguíneo durante exercício contínuo, devido ao equilíbrio entre a produção do lactato e a sua remoção da corrente sanguínea ${ }^{(24)}$.

Nos estudos em animais, a corrida em esteira rolante é bastante usada como modalidade de exercício. Esse ergômetro tem condições favoráveis, pois a mensuração da intensidade do esforço torna-se simples pelo controle da velocidade, da inclinação ou pela associação de ambos, além de poder usar o artifício do choque elétrico para estímulo. Sua utilização possui algumas inconveniências, como o elevado gasto com equipamento, a necessária realização de calibrações rotineiras da velocidade e a seleção de animais corredores ${ }^{(25)}$. Os testes aqui apresentados foram realizados em esteira rolante calibrada e revisada periodicamente, e não houve necessidade de utilizar choque elétrico como estímulo para a corrida dos animais.

Em 1993, Pillis et al. propuseram a aplicação de teste incremental e observação da resposta lactacidêmica para avaliação aeróbia de ratos corredores, baseando-se nos conceitos de limiar anaeróbio (Lan) sugerido para humanos ${ }^{(16)}$. Desse modo, os animais realizaram um teste caracterizado pelo aumento progressivo da velocidade, com estágios de cinco minutos e coletas de sangue ao final de cada carga, com posterior determinação do limiar anaeróbio por análise do comportamento exponencial da curva lactacidêmica. O limiar anaeróbio dos ratos foi obtido a $25 \mathrm{~m} \cdot \mathrm{min}^{-1}$, em concentração de lactato de $4 \mathrm{mM}^{(26)}$. Este achado é semelhante ao do presente estudo, no qual os ratos apresentaram média do ácido láctico após o segundo teste de 3,9mM, havendo redução do lactado durante o treinamento de $2 \mathrm{mM}$ uma vez que a média encontrada no primeiro teste, considerando-se os quatro grupos, foi de 5,9mM, demonstrando, assim, adaptação metabólica ao exercício proposto.

O estudo de Manchado et al. teve como objetivo padronizar a máxima fase estável de lactato em ratos corredores. Usaram ratos Wistar machos, adultos e sedentários, que foram submetidos a testes de corridas em intensidades equivalentes a 15, 20, 25, 30 e $35 \mathrm{~m} / \mathrm{min}$, sendo a sequência de velocidades distribuídas aleatoriamente, e os testes eram de corridas contínuas durante 25 minutos ou até a exaustão voluntária do animal. Foi realizada coleta de sangue a cada cinco minutos de exercício para determinação de curvas lactacidêmicas para cada uma das intensidades. Foi calculada a MFEL individual sendo conceituada como a mais alta intensidade de exercício na qual o aumento da lactacidemia foi igual ou inferior à $1 \mathrm{mmol} / \mathrm{L}$, do $10^{\circ}$ ao $25^{\circ}$ minuto de esforço. Os ratos corredores apresentaram MFEL em $20 \mathrm{~m} / \mathrm{min}$, com o metabólito permanecendo estável em $3,9 \pm 0,3 \mathrm{mmol} / \mathrm{L}^{(23)}$. A intensidade da Lan obtida nos resultados de Manchado et al. foi semelhante à descrita por Pillis et al., mesmo esse utilizando velocidades progressivas ${ }^{(26)}$. Os valores da concentração da lactacidemia sanguínea foi semelhante aos valores observados em protocolos experimentais de corridas com seres humanos, como por exemplo o estudo de Heck et al., que calculou a MFEL de 4mMol..(14). 
Existe na literatura um teste não invasivo sugerido por Monod e Scherer para avaliação aeróbia e anaeróbia através da potência crítica do exercício(27). Manchado et al., conforme as características e as aplicabilidades do mesmo, aplicou-o em esteira rolante, realizando um protocolo de padronização desse teste em ratos corredores ${ }^{(28)}$. Os ratos Wistar são submetidos a exercícios contínuos e exaustivos em intensidades supralimiar anaeróbio. São anotados a velocidade e tempo de exercício até a exaustão. Foi empregado um modelo matemático para estimar a máxima intensidade aeróbia (velocidade crítica $-V_{\text {crit }}$ ) e a capacidade de corrida anaeróbia (CCA); esse modelo foi capaz de ser adaptado para avaliação dos animais. $A V_{\text {crit }}$ foi identificada em 22,8 $\pm 0,7 \mathrm{~m} / \mathrm{min}$ e a CCA, 26,81 $\pm 2,6 \mathrm{~m}$. O resultado obtido da $V_{\text {crit }}$ comparado com a MFEL $(20 \mathrm{~m} / \mathrm{min})$ foi diferente, porém altamente correlacionados. Em relação a CCA, os autores sugerem novos estudos para efetivas conclusões (28). Como este tipo de avaliação não é precisa como a avaliação invasiva, esta metodologia não foi empregada no presente estudo.

O modelo simples e não invasivo do método de potência crítica tem algumas características que reduzem sua aplicabilidade, como a necessidade de exaustão dos animais durante os esforços para identificação do tempo limite de exercício. Como há na literatura um procedimento de avaliação com métodos não exaustivos, que podem ser utilizados em animais com diferentes condições fisiopatológicas e de treinamento, o estudo de Gobatto et al. teve como objetivo adaptar o protocolo não exaustivo de duplos esforços de Chassain ${ }^{(29)}$ para ava- liação aeróbia de ratos exercitados em esteiras rolantes ${ }^{(30)}$. O propósito do teste, inicialmente sugerido por Chassain, é a detecção do delta nulo de variáveis fisiológicas a partir de testes de duplos esforços em mesma intensidade. Para isso, ratos machos adultos realizaram quatro testes correntes em velocidades diferentes (10, 15, 20 e 25m.min-1), com intervalos de 48 horas entre eles. Os animais foram submetidos a dois esforços de cinco minutos em cada intensidade, com dois minutos de repouso entre eles e coleta de sangue para análise do lactato ao final do primeiro e segundo esforços ${ }^{(29,30)}$. Não empregamos a mesma metodologia, pois os animais testados no estudo aqui relatado eram submetidos a treinamento contínuo e de esforço máximo e não somente a esforço físico isolado, no qual o citado autor encontrou aumento progressivo do lactado devido ao modelo utilizado.

Os resultados do grupo controle (tabela 1) podem ser justificados pela idade e tempo de envelhecimento dos animais e falta de treinamento dos animais sedentários.

Os resultados aqui apresentados demonstraram que ratos submetidos a treinamento físico, de acordo com o protocolo testado, apresentam capacidade metabólica medida pelo ácido láctico, que demonstra aptidão física e treinamento adequado com aumento do esforço físico, sendo bem tolerado até 40 dias de treinamento em esteira rolante.

Todos os autores declararam não haver qualquer potencial conflito de interesses referente a este artigo.

\section{REFERÊNCIAS}

1. Nóbrega ACL. Fisiologia do Exercício. Rev SOCERJ 2000;4:19-23.

2. Trump ME, Heigenhauser GJ, Putman CT, Spriet LL. Importance of muscle phosphocreatine during intermittent maximal cycling. J Appl Physiol 1996;80:1574-80,

3. Spriet II, Howlett RA, Heigenhauser GJF. A human skeletal muscle during exercise. Med Sci Sports Exerc 2000;32:752-63

4. Katz A, Sahlin K. Regulation of lactic acid production during exercise. J Appl Physiol 1998;65:509-18.

5. Robergs R, Ghiasvand F, Parker D. Biochemistry of exercise-induced metabolic acidosis. Am J Physiol Regul Integr Comp Physiol 2004;287:R502-16.

6. Gaesser GA, Poole DC. The slow component of oxygen uptake kinetics in humans. Exerc Sports Sci Rev 1996;24:35-70.

7. Wasserman K, McLlory MB. Detecting the threshold of anaerobic metabolism. Am J Cardiol $1964 ; 14: 844-52$.

8. Manchado FB, Gobato CA. Máxima fase estável de lactato é ergonômetro depende em modelos experimentas utilizando ratos. Rev Bras Med Esporte 2006;12:259-62.

9. Ribeiro JP, Fielding RA, Hughes V, Black A, Bochese MA, Knuttgen HG. Heart rate break point may coincide with the anaerobic and not the aerobic threshold. Int J Sports Med 1985;6:220-4.

10. Ascensão AA, Santos P, Magalhães J, Oliveira J, Maia J, Soares J. Concentração sanguínea de lactato (CSL) durante uma carga constante a intensidade correspondente ao limiar aeróbio-anaeróbio em jovens atletas. Rev Paul Educ Fisi 2001;15:186-94.

11. Baptista RR, Oliveira LG, Figueiredo GB, Contieri JR, Loss JF, Oliveria AR. Limiar de lactato em remadores: comparação entre dois métodos de determinação. Rev Bras Med Esporte 2005;11:247-50.

12. Beneke R. Methodological aspects of maximal lactate steady state-implications for performance testing. Eur J Appl Physiol 2003;89:95-9.

13. Tehebur U, Busse MW, Braumann KM. Estimation of an individual equilibrium between lactate production and catabolism during exercise. Med Sci Sports Exerc 1993;25:620-7.

14. Heck H, Mader A, Hess G, Mucke S, Muller R, Hollmann W. Justication of the 4 mMol. l lactato threshold. Int J Sports Med 1985;6:117-30.

15. Svedahl K, Mclntosh BR. Anaerobic threshold: The concept and methods of measurement. Can J Appl Physiol 2003;28:299-323.
16. Sjodin B, Jacobs I, Karlsson J. Onset of blood lactate accumulation and marathon running performance. In J Sports Med 1981;2:23-6.

17. Crisafulli A, Tocco F, Pettau G, Caria M, Lorraia L, Melis F, Concu A. Detection of lactate threshold by including haemodynamic and oxygen extraction data. Physiol Means 2006;27:85-97.

18. Denadai BS, Ruas VDA, Figueira TR. Efeito da cadência de pedalada sobre as respostas metabólica e cardiovascular durante o exercício incremental e de carga constante em indivíduos ativos. Rev Bras Med Esporte 2005;11:286-90

19. Dwyer J, Bybee R. Heart rate indices of the anaerobic threshold. Med Sci Sports Exerc 1983;15:72-6.

20. Denadai BS, Balikian PJ. Relação entre limiar anaeróbio e "performance" no short triathlon. Rev Paul Edu Fisi 1995;9:10-5.

21. Granja PC, Pompeu, FAMS, Silva APRS. A acurácia da determinação do VO2máx e do limiar anaeróbio. Rev Bras Med Esporte 2005;11:167-71.

22. Ribeiro JP. Limiares metabólicos e ventilatórios durante o exercício. Aspectos fisiológicos e metodológicos. Arq Bras Cardiol 1995;64:171-81.

23. Manchado FB, Gobatto CA, Contarteze RVL, Papoti M, Mello MAR. Maximal lactate steady state in running rats. J Exer Physiol online 2005;8:29-35.

24. Beneke R. Maximal lactate steady state concentration (MLSS): experimental and modeling approaches. Eur J Appl Physiol. 2003;88(4-5):361-9,

25. Gobatto CA, Mello MAR, Gobatto FBM, Papoti M, Voltarelli FA, Contarteze RV, Araujo GG. Avaliações fisiológicas adaptadas a roedores: aplicações ao treinamento em diferentes modelos experimentais. Rev Mackenzie Edu Fisi Esp 2008;7:137-47.

26. Pillis W, Zarzeczny R, Langfort J, Kaciuba-Uscilko H, Nazar K, Wojtyna J. Anaerobic threshold in rats. Comp Biochem Physiol 1993;106A:285-9.

27. Monod H, Scherer J. The work capacity of a synergic muscular group. Ergonomics 1965;8:329-38.

28. Manchado FB, Contarteze RVL, Gobatto CA, Papotti M, Mello MAR. Critical velocity and anaerobic running capacity determination of running rats. Med Sci Sports Exer 2006;38:516.

29. Chassain A. Méthode d'appréciation objetive de la tolérance de l'organisme á l'effort: application á la mensure des puissances de la frequence cardiaque et de la lactatémie. Science \& Sports 1986;1:41-8

30. Gobatto CA, Manchado FB, Contarteze RVL, Mello MAR. Double bouts test for non-exhaustive aerobic evaluation of running rats. Med Sci Sports Exer (suppl) 2006;35:517. 